GYNAECOLOGICAL CANCER

\title{
Steps towards effective gynaecological cancer screening Usha Menon
}

PapSEEK, a novel liquid-based cytology test, enabled the highly specific detection of endometrial and ovarian cancer in a retrospective study. I discuss potential applications of this tool, both for cancer screening of asymptomatic populations, and for the early diagnosis of cancer in symptomatic women.

Refers to Wang, Y. et al. Evaluation of liquid from the Papanicolaou test and other liquid biopsies for the detection of endometrial and ovarian cancers. Sci. Trans/ Med. 10, eaap8793 (2018).

There are two issues facing the gynaecological cancer community - rising endometrial cancer rates in response to mounting obesity and longevity and the continued diagnosis of ovarian cancer at an advanced stage with high case fatality ratio, despite concerted worldwide efforts to diagnose it earlier. Current screening tests for both cancers lack specificity. To address this, Wang and collaborators ${ }^{1}$ refined the proof-of-principle test that they had previously developed to detect cell free tumour DNA in liquid-based cytology (Pap) specimens from patients with endometrial or ovarian cancer ${ }^{2}$.

In their new test, PapSEEK, they included multiplex PCR to detect mutations in 18 genes and assays to detect aneuploidy. In a retrospective case-control study of 1,658 individuals, sensitivity for the detection of endometrial cancer was $81 \%$ and for ovarian cancer $33 \%$. By using additional biospecimens (intrauterine samples obtained with a Tao brush for endometrial cancer and plasma samples for ovarian cancer) they were able to increase the sensitivity to $93 \%$ and $63 \%$, respectively. The PapSEEK test had high specificity ( 99\%), a key requirement for cancer screening. However, in this respect, it needs to be noted that control women were much younger (median ages 34 and 29 of the Pap and Tao brush cohorts respectively) than the cancer patients (median ages 68 and 57 of endometrial and ovarian cancer cohort respectively). With increasing age, the possibility of detecting mutations is likely to increase, owing to the increased incidence of both cancer-related mutations and other unrelated conditions. In addition the control cohorts, only included women who had not been diagnosed with any cancer. Population screening, however, would involve all individuals, including those with other cancers. The next steps in the evaluation of PapSEEK would be to assess performance characteristics in a prospective cohort study, the design of which would depend on the specific application - screening of asymptomatic populations or early detection of cancer in symptomatic women.

With endometrial cancer, although the incidence is rising, over three-quarters of women survive for ten years or more and most are diagnosed at an early stage ${ }^{3,4}$. This mostly favourable disease course, together with the low specificity of the currently available transvaginal ultrasound test ( $85 \%$ for endometrial thickness cutoff of $5 \mathrm{mms}^{5}$ ) makes endometrial cancer screening in the general population difficult to justify. The use of PapSEEK, however, would not require the implementation of an entirely new screening endometrial cancer screening programme; it would only 
involve an additional highly specific test on the liquid cytology samples already collected during cervical cancer screening. If such tests were limited to women aged $>45$ years - the age at which the incidence of endometrial cancer begins to rise $^{3,4}$-then it might prove to be cost-effective, especially as there has been a small but steady increase $\left(1.6 \%\right.$ per year $\left.{ }^{3,4}\right)$ in uterine cancer mortality rates over the past decade. This test could become a true game changer if, in the future, it were to include unique DNA signatures associated with progression of precursor lesions to malignancy.

In ovarian cancer, diagnosis at predominantly advanced stages, high casefatality ratios, and the lack of a definitive mortality reduction with current screening strategies has fuelled the hunt for more effective approaches. The use of PapSEEK on liquid cytology specimens would inevitably include ovarian cancer screening. Although the sensitivity is low $(33 \%)^{1}$, this strategy would improve the cost-benefit ratio of testing cervical cytology samples. Prospective cohort studies are needed to evaluate the cost effectiveness of increasing sensitivity by testing additional plasma and/or intra-uterine samples. Given that high grade serous ovarian cancers may spend on average $>4 \mathrm{yrs}$ as in situ, stage I, or stage II cancers 6 , it maybe possible to decrease screening frequency from annual to once in two to three years. Finally randomised controlled trials would need to be undertaken to evaluate the impact of PapSEEK on ovarian cancer mortality. As PapSEEK for detection of ovarian cancer is based on passage of tumour DNA from the tubes and ovaries into the lower genital tract, it cannot be used in women where this has been interrupted by common procedures such as tubal ligation, hysterectomy, endometrial ablation, and possibly intrauterine device placement. A limitation with plasma samples is that circulating cell-free DNA concentrations vary depending on how the blood is processed and the interval of time from collection to analysis.

In the randomised controlled trial of ovarian cancer screening (UKCTOCS), multimodal screening of women in the general population using serial serum CA-125 levels as a first-line test resulted in high sensitivity ( 85\%), significant increase in diagnosis of early stage invasive epithelial ovarian, tubal, and peritoneal cancers ( $36 \%$ versus $24 \% ; p=0.00013)$ and a nonsignificant $(p=0 \cdot 10)$ reduction in ovarian cancer mortality compared to no screening ${ }^{7}$. The multimodal strategy required repeat CA-125 testing and transvaginal ultrasonography at 6-12 week intervals in those with abnormal first-line test results, to ensure high specificity and reduce the number of unnecessary operations ${ }^{8}$. A highly specific second-line test, such as PapSEEK using Tao and Pap brush and plasma DNA samples, could substantially shorten the time from first-line testing to diagnosis in this strategy. This improvement would be particularly relevant if a definitive mortality reduction was found in 2019 at the end of extended follow-up of the UKCTOCS cohort.

Unlike in the general population, the combined screening approach involving the Tao brush and cervical cytology could be of immediate relevance to women at high risk of developing endometrial cancer (for example, those with Lynch syndrome). These women are offered endometrial cancer screening - often involving annual endometrial sampling - following a discussion of pros and cons. In this context, less invasive testing of Pap samples using PapSEEK could be an attractive and more acceptable alternative for some women. In women at high risk of ovarian cancer (such as those with BRCA mutations), there is no clear consensus 
regarding screening. Risk-reducing salpingo-oophorectomy is the recommended approach. Evaluation of PapSEEK in samples taken before surgery from such patients who have a $6 \%$ prevalence of unexpected adnexal neoplasia ${ }^{9}$ could provide preliminary data on performance characteristics. In this population, specificity is likely to be lower than in the general population, because these women are likely to develop other cancers (for example, breast, pancreas, or bowel cancer); however, any associated unnecessary surgery is less of an issue in this setting.

Another area in which PapSEEK could be of great value is in the triage of women with ovarian cancer 'alert' symptoms, such as persistent abdominal or pelvic pain, bloating or increase in abdominal girth or feeling full quickly. In these women, disease burden is higher than in asymptomatic women and hence, sensitivity might be similar to that noted in the study (63\%) discussed herein ${ }^{1}$. Both plasma and liquid cytology samples can be collected easily at primary care centres, and positive results could enable fast tracking to specialist gynaecological oncology centres. In older women, positive PapSEEK results might be due to other cancers. Hence if findings are normal on transvaginal ultrasonography, CT imaging of chest, abdomen, and pelvis and mammography are required to identify the source of the detected genetic alterations ${ }^{8}$. In the future, however, methylation-profiling of the cell-free tumour DNA could provide insights into the tissue of origin and thus tumour location ${ }^{10}$.

Finally, the use of PapSEEK on liquid cytology specimens might be a useful tool in health-care settings where women with postmenopausal bleeding initially present to a primary care physician and not a gynaecologist. The current management of these women requires transvaginal ultrasonography and, in the absence of a thin distinct endometrial echo, sonohysterography, office hysteroscopy, or endometrial sampling. The value of PapSEEK when these patients are admitted in specialist health-care settings is harder to define. While PapSEEK using a Pap and Tao brush (93\%) has similar sensitivity to endometrial sampling $(90-100 \%)^{7}$, the latter allows for histological assessment, which is a key to planning further management.

In conclusion, prospective evaluation in multiple patient settings is required before PapSEEK can be used in routine clinical settings. Nonetheless, PapSEEK remains a promising test with the potential to improve screening for and early detection of gynaecological cancers.

Usha Menon is at the Gynaecological Cancer Research Centre, Women's Cancer, Institute for Women's Health, University College London, UK.

email: u.menon@ucl.ac.uk

\section{References}

1. Wang, Y. et al. Evaluation of liquid from the Papanicolaou test and other liquid biopsies for the detection of endometrial and ovarian cancers. Sci. Trans/ Med. 10, eaap8793 (2018).

2. I. Kinde, C. et al. Evaluation of DNA from the Papanicolaou test to detect ovarian and endometrial cancers. Sci. Transl. Med. 5, 167ra4 (2013).

3. Surveillance, Epidemiology, and End Results (SEER) Program Cancer Stats

Facts. https://seer.cancer.gov/statfacts/html/corp.html Accessed May 2018 
4. Cancer Research UK Cancer Statistics http://www.cancerresearchuk.org/healthprofessional/cancer-statistics/statistics-by-cancer-type/uterine-cancer Accessed May 2018.

5. Jacobs I, et al. Sensitivity of transvaginal ultrasound screening for endometrial cancer in postmenopausal women: a case-control study within the UKCTOCS cohort. Lancet Oncol. Jan;12(1):38-48 (2011)

6. Brown PO, Palmer C. The preclinical natural history of serous ovarian cancer: defining the target for early detection. PLoS Med. 6(7):e1000114. (2009)

7. IJ. Jacobs et al. Ovarian cancer screening and mortality in the UK Collaborative Trial of Ovarian Cancer Screening (UKCTOCS): A randomised controlled trial. Lancet 387, 945-956 (2016).

8. U. Menon et al. Risk algorithm using serial biomarker measurements doubles the number of screen-detected cancers compared with a single-threshold rule in the United Kingdom Collaborative Trial Of Ovarian Cancer Screening. J. Clin. Oncol. 33, 2062-2071 (2015).

9. Powell $\mathrm{CB}$. Risk reducing salpingo-oophorectomy for BRCA mutation carriers: twenty years later. Gynecol Oncol.;132(2):261-3. (2014)

10. Kang S, et al. Cancer Locator: non-invasive cancer diagnosis and tissue-of-origin prediction using methylation profiles of cell-free DNA. Genome Biol 18:53. (2017) 11. van Hanegem N, Prins MM, Bongers MY, Opmeer BC, Sahota DS, Mol BW, Timmermans $A$. The accuracy of endometrial sampling in women with postmenopausal bleeding: a systematic review and meta-analysis. Eur J Obstet Gynecol Reprod Biol. 197:147-55. (2016)

\section{Acknowledgements}

The work of U.M. is supported by the NIHR University College London Hospitals Biomedical Research Centre.

\section{Conflict of interest}

U.M. has stock ownership awarded by her employer (University College London) in and has received research funding from Abcodia Pvt $L t d$, a company with an interest in screening and early detection of cancer. 